\title{
Association of MiR-149 with Nutritional Risk Assessment and Postoperative Complications of Patients with Colorectal Cancer
}

\author{
Lin Lin ${ }^{1}$, Feipeng $\mathrm{Xu}^{2}$, Caijin Zhou², Zhenhao Quan² and Haiping Jiang1,* \\ ${ }^{1}$ Department of General Surgery, the First Affiliated Hospital, Jinan University, Guangzhou, China \\ ${ }^{2}$ Department of Gastrointestinal Surgery, the Affiliated Hospital of Guangdong Medical University, Guangzhou, China
}

* Corresponding author: Haiping Jiang, Department of General Surgery, the First Affiliated Hospital, Jinan University, Guangzhou, China. Tel: 86013392692225; Email: wcwkll@126.com

Received 2021 June 18; Revised 2021 November 06; Accepted 2021 November 28.

\begin{abstract}
Background: MiRNAs play an important role in the development of colorectal cancer (CRC); however, there is little evidence of miRNAs in the screening of the nutritional risks of patients with CRC.

Objectives: This study aimed to explore the role of preoperative miR-149 in nutritional screening of patients with CRC, and its associations with clinicopathological characteristics and postoperative complications of patients with CRC.

Methods: The associations of serum miR-149 with clinicopathological characteristics and postoperative complications of patients were analyzed in this study. The receiver operating characteristic curves were plotted with miR-149 as the test variable, the grouping results of the patients with nutritional risks (total NRS2002 score $\geq 3$ points), and no nutritional risks (total NRS2002 score $<3$ points) based on the preoperative NRS2002 score as the state variables. The consistency of miR-149 and NRS2002 in the nutritional screening of CRC was analyzed using the Kappa test.

Results: MiR-149 was low in patients with CRC. There was a statistically significant difference in the miR-149 expression among patients with different tumor diameters and TNM stages in the two groups. The preoperative total NRS2002 score of CRC patients was $<3$ (without nutritional risks) in 271 cases, and $\geq 3$ (with nutritional risks) in 129 cases. The sensitivity and specificity of miR-149 in the diagnosis of nutritional risks were $76.74 \%$ and $84.50 \%$, respectively. The Kappa value was 0.622 with miR $-149=3.095$ as the critical value.

Conclusion: MiR-149 can reflect the preoperative nutritional status of patients with CRC, and miR-149=3.095 can be used as the cut-off point for nutritional risk screening of patients with CRC, which is an important index for assessing the nutritional risk in the preoperative period. The expression of miR-149 has a certain association with postoperative complications.
\end{abstract}

Keywords: Colorectal cancer, Complications, MiR-149, Nutritional risk

\section{Background}

Colorectal cancer (CRC) is one of the most common causes of malignant tumor-related deaths in the world (1), and it is the most common malignancy of the digestive system. There are more than 1.2 million new cases and no less than 600,000 deaths of CRC every year, and they both show increasing trends year by year (2). Among all cancers in the world, the morbidity rate of CRC ranks third, and its mortality rate ranks fourth in the world (3). Many CRC patients will be misdiagnosed, and less than $40 \%$ of the patients can be diagnosed early. As a result, more than $50 \%$ of the CRC patients have missed the best opportunity of diagnosis and treatment, and some have already been in TNM stage III+IV when treated, with a 5-year survival rate of far lower than $40 \%$ (4). Therefore, it is needed to actively find better markers for early detection and adjuvant therapy of CRC, which has extraordinary value for altering the prognosis of patients.

In the field of gastrointestinal surgery in recent years, the nutritional problem of surgical patients in the preoperative period has aroused widespread concern, and nutritional status has become a key factor for the postoperative recovery of patients. It is emphasized by the European Society of Parenteral and Enteral Nutrition that patients with preoperative nutritional risks may suffer from poor prognoses (5). Malnutrition has a high incidence rate in patients with gastrointestinal malignancy, which directly leads to poor postoperative incision healing, pulmonary infection, and other complications; therefore, the length of hospital stay will be prolonged, and the treatment expenses will be increased. A nutritional crisis is also an important adverse factor for the long-term prognosis of CRC patients. Nutrition Risk Screening 2002 (NRS2002) is a widely-used screening method for nutritional risks around the world. However, NRS2002 has complicated scoring procedures and requires much time and effort; moreover, it is susceptible to the subjective factors of patients, greatly limiting its popularization (6).

Micro ribonucleic acids (miRNAs) are a class of non-coding RNAs of relatively small molecular weights, with about 20-25 nucleotides in molecular size (7). A study has shown that a large number of miRNAs are dysregulated in tumors and widely involved in many biological processes of tumor cells

Copyright (C) 2021, Author(s). This is an open-access article distributed under the terms of the Creative Commons Attribution-NonCommercial 4.0 International License (http://creativecommons.org/licenses/by-nc/4.0/) which permits copy and redistribute the material just in noncommercial usages, provided the original work is properly cited 
(8). MiR-149, a new miRNA discovered recently, is located in intron 1 of Glypican-1 at chromosome 2q37.3. In breast cancer, gastric cancer, and nonsmall cell lung cancer, miR-149 can serve as a tumor suppressor miRNA in epigenetic silencing and play a role as a cancer suppressor gene $(7,9)$. There is a study showing that miR-149 can inhibit the proliferation of CRC cells, thereby inhibiting the occurrence and development of CRC (10).

\section{Objectives}

Currently, there are few studies on whether preoperative miR-149 is accurate in screening nutritional risks of CRC patients in China and on the optimal value of diagnosis. In the present study, with NRS2002 as the golden standard for the diagnosis of nutritional risks of CRC patients, the significance of miR-149 in the screening of nutritional risks of patients with CRC was explored, and the associations of miR-149 with clinicopathological characteristics and postoperative complications of patients with CRC were retrospectively analyzed.

\section{Methods}

\subsection{General data}

The clinical data of 400 patients diagnosed with CRC via postoperative pathology and undergoing operative treatment were collected in this study. The inclusion criteria were: (1) patients treated with the elective radical operation of CRC, (2) those diagnosed with CRC via postoperative histopathology, (3) those who could generally communicate, (4) those with complete clinical data, (5) those without other factors causing malnutrition or severe infectious diseases, and (6) those without receiving operative treatment or chemoradiotherapy of CRC before admission. On the other hand, (1) patients with multi-source malignant tumors, (2) those who had undergone radiotherapy, chemotherapy, or other treatments of CRC before this operation, (3) those undergoing emergency operation, (4) those with distant metastasis according to preoperative examination, (5) preoperative deaths, (6) those with incomplete clinical data, or (7) those with other factors causing malnutrition or severe infectious diseases were excluded from the study. During the same period, 200 healthy people were selected from the physical examination center as the healthy control group. The study protocol was approved by the Ethics Committee of the First Affiliated Hospital, Jinan University (017-SD-30-ME-N0.102), Guangzhou, China. Signed written informed consents were obtained from all participants before the study.

\subsection{NRS2002 screening}

The nutritional screening was performed for all patients by specially-trained investigators $24 \mathrm{~h}$ after hospitalization, and the results were assessed using a standard unified questionnaire. The scoring criteria for nutritional status included 0 (normal nutritional status), 1 (weight loss $>5 \%$ within 3 months or decline in food intake by $25.50 \%$ one week before hospitalization), 2 (weight loss $>5 \%$ within 2 months/decline in food intake by $50 \%-75 \%$ one week before hospitalization, or body mass index[BMI] $<18.5-20.5 \mathrm{~kg} / \mathrm{m}^{2}$ ), and 3 (weight loss $>5 \%$ within 1 month/decline in food intake by $75 \%$ $100 \%$ one week before hospitalization, or BMI $<18.5$ $\mathrm{kg} / \mathrm{m}^{2}$ ). In addition, the score of disease severity was 2 for preoperative patients with CRC, and the score of age $\geq 70$ years old was 1 . The total score is the sum of the scores of nutritional status, disease severity, and age. The total score of $\geq 3$ indicates nutritional risks, while the score of $<3$ indicates no nutritional risks (11). In addition to the questionnaire survey, fasting venous blood was drawn from patients enrolled the next morning after admission, and serum albumin (ALB) was detected to assess the objective nutritional index.

\subsection{Quantitative reverse transcription-polymerase chain reaction}

The total RNA was extracted from tissues using TRIzol reagent (Invitrogen, Carlsbad, CA, USA), and the isolated RNA was quantified using NanoDrop 2000 (Thermo Fisher Scientific, Inc., Waltham, MA, USA). Following that, RNA was reversely transcribed into DNA using PrimeScript ${ }^{\mathrm{TM}}$ RT Master Mix (TaKaRa Bio., Inc., Tokyo, Japan), and qPCR was performed using TBGreen ${ }^{\circledR}$ Premix Ex Taq ${ }^{\mathrm{TM}}$ II (TaKaRa Bio., Inc., Tokyo, Japan) on CFX-96 quantitative reverse transcription-polymerase chain reaction (qRT-PCR) system (Bio-Rad Technologies, Inc., Hercules, CA, USA). The thermal cycling conditions are as follows: initial denaturation at $95^{\circ} \mathrm{C}$ for $30 \mathrm{sec},\left(95^{\circ} \mathrm{C}\right.$ for $5 \mathrm{sec}$ and $60^{\circ} \mathrm{C}$ for $\left.30 \mathrm{sec}\right) \times 40$ cycles, with $\mathrm{U} 6$ as an internal reference. The primer sequences are as follows: miR-149 F: 5'-GGCTCTGGCTCCGTGTCTT-3', R: 5'-CAGTGCAGGGTCCGAGGTATT-3'. U6 F: 5'CTCGCTTCGGCAGCACA-3', R: 5'-AACGCTTCACGAA TTTGCGT-3'. The relative expression level of miR-149 was calculated by the $2-\Delta \Delta \mathrm{Cq}$ method.

\subsection{Statistical analysis}

Statistical Product and Service Solutions (SPSS software; version 22.0; IBM, Armonk, NY, USA) was used for data analysis. Measurement data were expressed as mean $\pm S D$, and a t-test was used for comparison. Enumeration data were tested by $\chi^{2}$ test. The receiver operating characteristic (ROC) curve was plotted, and the area under the curve (AUC), sensitivity, and specificity were calculated. The consistency of miR-149 and NRS2002 in evaluating the nutritional risk of CRC patients was detected using the Kappa test. A p-value less than 0.05 was considered statistically significant. 


\section{Results}

\subsection{Serum miR-149 was lowly expressed in CRC patients}

The results of qRT-PCR showed that the expression of serum miR-149 significantly declined in 400 CRC patients, compared to that in 200 healthy controls (Figure 1). It can be observed that serum miR-149 is lowly expressed in CRC patients, and miR149 can play a role as a cancer suppressor gene in the occurrence and development of CRC.

4.2. Associations between miR-149 expression and clinicopathological characteristics of patients

To explore whether miR-149 has statistical

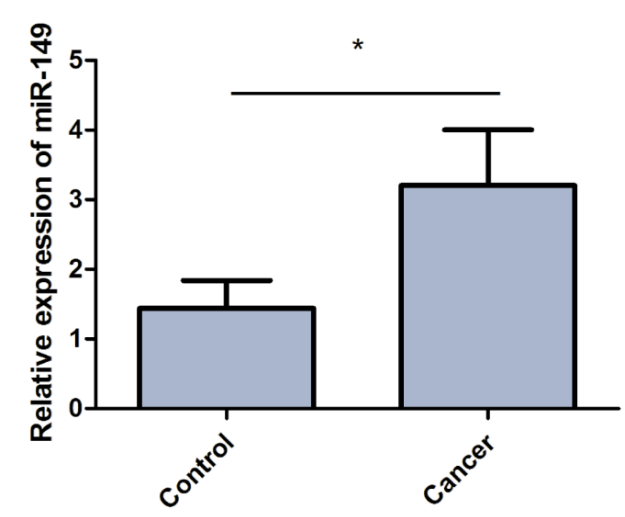

Figure 1. Serum miR-149 was lowly expressed in CRC patients. The results of qRT-PCR showed that the expression of serum miR-149 significantly declined in CRC patients, compared to that in healthy controls $(\mathrm{P}<0.05)$. differences in terms of the clinicopathological characteristics of CRC patients, the patients were grouped based on the median expression of miR-149. It was found that miR-149 was not related to the nutritional risk, gender, age, tumor site, tumor infiltration depth, and presence or absence of distant metastasis ( $>>0.05)$; however, it showed a statistically significant difference among patients with different tumor sizes and TNM stages $(\mathrm{P}<0.05)$. In the low miR-149 group, the proportion of patients with a tumor diameter $>4 \mathrm{~cm}$ was obviously higher than that with a tumor diameter $\leq 4 \mathrm{~cm}$, while the proportion of patients in TNM the stage III + IV was obviously higher than that in stage I + II (Table 1). These results indicate that the expression level of miR-149 can affect the tumor diameter and TNM stage of CRC patients.

\subsection{Sensitivity and specificity of miR-149 in nutritional risk assessment}

ALB is the most important protein in human plasma, which is able to keep the nutrition and osmotic pressure of the body and reflect the nutritional status of patients to a certain extent. In this study, the ALB level was detected for objective verification. The results revealed that the level of serum ALB was $<35$ $\mathrm{g} / \mathrm{L}$ in patients with an NRS2002 score of $\geq 3$, while it was $\geq 35 \mathrm{~g} / \mathrm{L}$ in patients with an NRS2002 score of $<3$. Among the 400 subjects, the NRS2002 score was $<3$ (without nutritional risks) in 271 cases, and $\geq 3$ (with nutritional risks) in 129 cases.

\begin{tabular}{|c|c|c|c|c|c|}
\hline Variable & $\mathbf{n}$ & $\begin{array}{c}\text { Low level } \\
(\mathrm{n}=200)\end{array}$ & $\begin{array}{c}\text { High level } \\
(\mathrm{n}=200)\end{array}$ & $\chi^{2}$ & $\mathbf{P}$ \\
\hline \multicolumn{6}{|l|}{ Gender } \\
\hline Male & 206 & 112 & 94 & 3.243 & 0.089 \\
\hline Female & 194 & 88 & 106 & & \\
\hline \multicolumn{6}{|l|}{ Age } \\
\hline$<60$ & 159 & 83 & 76 & 0.511 & 0.54 \\
\hline$\geq 60$ & 241 & 117 & 124 & & \\
\hline \multicolumn{6}{|c|}{ Tumor site } \\
\hline Colon & 224 & 111 & 113 & 0.041 & 0.92 \\
\hline Rectum & 176 & 89 & 87 & & \\
\hline \multicolumn{6}{|c|}{ Tumor diameter } \\
\hline$\leq 4 \mathrm{~cm}$ & 186 & 70 & 116 & 21.264 & $<0.001$ \\
\hline$>4 \mathrm{~cm}$ & 214 & 130 & 84 & & \\
\hline \multicolumn{6}{|c|}{ TNM staging } \\
\hline $\mathrm{I}+\mathrm{II}$ & 185 & 77 & 108 & 9.664 & 0.003 \\
\hline III +IV & 215 & 123 & 92 & & \\
\hline \multicolumn{6}{|c|}{ Depth of infiltration } \\
\hline $\mathrm{T} 1+\mathrm{T} 2$ & 169 & 89 & 80 & 0.83 & 0.418 \\
\hline $\mathrm{T} 3+\mathrm{T} 4$ & 231 & 111 & 120 & & \\
\hline \multicolumn{6}{|c|}{ Lymphatic metastasis } \\
\hline No & 206 & 101 & 105 & 0.16 & 0.764 \\
\hline Yes & 194 & 99 & 95 & & \\
\hline \multicolumn{6}{|c|}{ Distant metastasis } \\
\hline No & 167 & 79 & 88 & 0.833 & 0.417 \\
\hline Yes & 233 & 121 & 112 & & \\
\hline
\end{tabular}




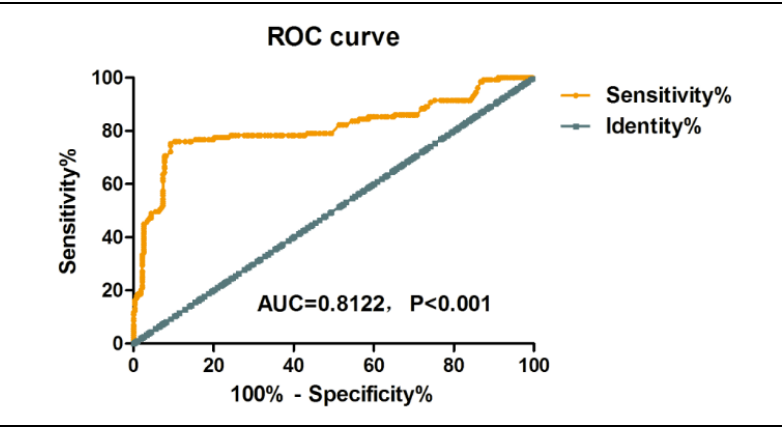

Figure 2. Sensitivity and specificity of miR-149 in nutritional risk assessment. The AUC was 0.8122 (95\% CI: 0.7597-0.8648) $(\mathrm{P}<0.001)$. The sensitivity and specificity of miR-149 in the diagnosis of nutritional risks are $76.74 \%$ and $84.50 \%$, respectively, when its cut-off point was 3.095 .

To further explore the sensitivity and specificity of miR-149 in assessing nutritional risks, the ROC curve was plotted with miR-149 as the test variable. The results manifested that the AUC was 0.8122 (95\% CI: 0.7597-0.8648) $(\mathrm{P}<0.001)$. The sensitivity and specificity of miR-149 in the diagnosis of nutritional risks were $76.74 \%$ and $84.50 \%$, respectively, when its cut-off point was 3.095 (Figure 2). The results suggest that miR-149 is a potential biomarker for assessing the nutritional risk of CRC patients.

4.4. Analysis of the consistency of miR-149 and NRS2002 in the diagnosis of nutritional risk of CRC patients

According to the ROC curve of miR-149 in evaluating the nutritional risk of CRC patients, the optimal cut-off point of miR-149 was 3.095, based on which the patients were divided into a high miR-149 group ( $n=287$, miR-149 $\geq 3.095$ ) and low miR-149 group ( $\mathrm{n}=113$, miR-149<3.095). The consistency of miR-149 and NRS2002 in the diagnosis of nutritional risk of CRC patients was analyzed. The results manifested that the Kappa value was 0.622 with miR$149=3.095$ as the critical value $(\mathrm{P}<0.001)$ (Table 2$)$. To sum up, miR-149 is in good consistency with NRS2002.

\begin{tabular}{|c|c|c|}
\hline \multirow{2}{*}{ NRS2002 Score } & \multicolumn{2}{|c|}{ Cut-off value of miR-149 } \\
\hline & $<3.095(n=287)$ & $\geq 3.095(n=113)$ \\
\hline$<3(n=271)$ & 247 & 24 \\
\hline$\geq 3(n=129)$ & 40 & 89 \\
\hline Kappa & \multicolumn{2}{|c|}{0.622} \\
\hline $\mathbf{P}$ & \multicolumn{2}{|c|}{$<0.001$} \\
\hline
\end{tabular}

\begin{tabular}{lcccc}
\hline Table 3. Relationship between miR-149 and postoperative complications & & \\
\hline Variable & $\mathbf{n}$ & $\begin{array}{c}\text { Low level } \\
\text { (n=287) }\end{array}$ & $\begin{array}{c}\text { High level } \\
\text { (n=113) }\end{array}$ & $\boldsymbol{\chi}^{\mathbf{2}}$ \\
\hline Anastomotic bleeding & 22 & 18 & 4 & 1.164 \\
Anastomotic fistula & 35 & 27 & 8 & 0.339 \\
Intestinal obstruction & 15 & 12 & 3 & 0.55 \\
Lung infection & 19 & 17 & 2 & 0.523 \\
None & 309 & 213 & 96 & 3.091 \\
\hline
\end{tabular}

\subsection{Association between miR-149 and postoperative complications}

To further clarify whether the preoperative expression of serum miR-149 can predict the occurrence of postoperative complications, 400 patients undergoing CRC surgery were followed up and observed. It was found that postoperative complications occurred in 91 patients.

The total incidence rate of complications (anastomotic bleeding [ $\mathrm{n}=22]$, anastomotic fistula $[\mathrm{n}=35]$, intestinal obstruction $[\mathrm{n}=15]$, and pulmonary infection [ $n=19]$ ]) was $22.75 \%$. With miR-149=3.095 as the cut-off value, whether there is a correlation between the preoperative miR-149 level and postoperative complications was analyzed. The results showed that $17(15.04 \%)$ cases had postoperative complications in a high miR-149 group, and $74(25.78 \%)$ cases had postoperative complications in a low miR-149 group. According to the statistical analysis, the association between miR149 expression and the incidence rate of postoperative complications was statistically significant $(\mathrm{P}<0.05)$ (Table 3$)$.

The above findings indicate that the high expression of miR-149 can lower the incidence of postoperative complications.

\section{Discussion}

MiRNAs play regulatory roles in a variety of biological processes, including cell differentiation, proliferation, migration, invasion, apoptosis, and cell cycle progression. Moreover, they can regulate gene expression and various cellular mechanisms at the post-transcriptional level and serve as important factors for carcinogenesis (12). Studies have demonstrated that miRNAs can be quickly detected non-invasively, and they are extremely stable under various conditions in the laboratory. Link et al. (13) found via RT-PCR and microarray analysis that the expressions of miR-21 and miR-106a are significantly up-regulated in the feces of CRC patients. Wu et al. 
(14) detected miR-21a and miR-92a in tissues and feces of the CRC patients and normal controls using qRT-PCR and found that the CRC patients have increased expressions of miR-21 and miR-92a in tissues and feces. The expression of miR-92a can help distinguish patients with CRC or adenoma and healthy controls or even patients with low-risk polyps. Moreover, miRNAs can be used as a new prognostic biomarker. Lv et al. (15) studied and found that high expression of miR-155 before the operation is closely associated with late stage and metastasis, while its high expression after the operation is associated with recurrence and metastasis, suggesting that miR-155 can serve as a prognostic marker for tumor-free survival.

MiR-149 is a kind of non-coding RNA widely concerned in recent years, with $23 \mathrm{nt}$ in length, which can inhibit the translation of target mRNA by binding to its 3'UTR and exerting a post-transcriptional regulatory effect. There is increasing evidence that miR-149, as a proto-oncogene or a cancer suppressor gene, is involved in regulating the occurrence and development of breast cancer, gastric cancer, liver cancer, and lung cancer; moreover, its abnormal expression enhances the malignant biological behaviors of tumor cells, such as proliferation, invasion, and migration (16-18). Xu et al. (19) proved in CRC HCT116, LoVo, and SW480 cell lines that overexpression of miR-149 leads to the decline in CRC cell invasion and migration, thereby suppressing the malignant biological behaviors of CRC cells. These conclusions are similar to the results in this study that the expression of serum miR-149 evidently declined in CRC patients, and it could affect the tumor size and TNM stage of patients.

A large number of research reports have shown that patients with malignant tumors often suffer from a nutritional crisis. The nutritional status of patients is closely related to tumor progression and poor clinical prognosis. Active preoperative nutritional intervention can reduce nutritional risks and poor prognosis (20,21). A multi-country multi-center clinical trial confirmed that the NRS2002 score has a close correlation with the length of hospital stay, complications, and death rate of patients undergoing gastrointestinal surgery (22). Schwegler et al. (23) reported that the NRS2002 score can be used to predict the incidence rate of postoperative complications, and even exhibits a positive trend in predicting postoperative mortality. There is also a study showing that the application of NRS2002 in the screening of nutritional risk of CRC patients can provide a reasonable and effective basis for clinical nutritional support (24). In this study, the NRS2002 score was given to all patients. In nutritional risk screening with miR-149, the AUC was 0.8122 , and the sensitivity and specificity of miR-149 in the diagnosis of nutritional risks were $76.74 \%$ and $84.50 \%$, respectively, when its cut-off point was 3.095. It can be observed that miR-149 has high accuracy in the preoperative nutritional screening of CRC patients. The patients were divided into two groups based on the preoperative miR-149 level. In the Kappa test, the Kappa value was 0.622 with miR-149=3.095 as the cut-off value $(\mathrm{P}<0.001)$. It was confirmed that miR149 is in good consistency with NRS2002, and can be used to assess the nutritional risks of CRC patients. In addition, the incidence rate of postoperative complications was lower in patients with miR-149 $\geq 3.095$ than that in patients with miR-149 <3.095, which had a statistical significance. The novelty of this study was that it was the first time to explore the role of preoperative miR-149 in the nutritional screening of patients with CRC and also investigate its associations with clinicopathological characteristics and postoperative complications. However, limitations still existed in our present study. The sample was not big enough to support our final conclusions with very strong evidence. In our future study, it will be planned to enlarge the sample to employ more eligible patients to obtain more powerful data to support our findings. Furthermore, some molecular experiments will be conducted to explore the potential mechanism in which miR-149 works in this process.

Many patients with CRC have a nutritional crisis; therefore, preoperative nutritional risk assessment and nutritional intervention for patients with malnutrition are extremely important in colorectal surgery, which can enable more CRC patients to receive better treatment and raise the survival rate of patients with a low miR-149 expression.

\section{Conclusion}

Serum miR-149 is lowly expressed in CRC patients. Moreover, MiR-149 is in good consistency with NRS2002 in nutritional risk assessment of CRC and is also associated with postoperative complications of CRC patients. MiR-149 is expected to become a new marker for CRC.

\section{Acknowledgments}

None.

\section{Footnotes}

Authors' contributions: LL and HJ designed the study. LL, FX, and HJ performed the experiments. CZ and ZQ collected and analyzed the data. LL, CZ, and HJ prepared the manuscript. All authors read and approved the final manuscript.

Ethical Approval: This study was approved by the Ethics Committee of the First Affiliated Hospital, Jinan University (017-SD-30-ME-N0.102), Guangzhou, China. Funding/Support: This study did not receive any funding in any form. 
Conflicts of Interest: All authors declare that they have no conflict of interest.

Informed Consent: Signed written informed consents were obtained from all participants before the study.

\section{References}

1. Jafarian AH, Forooshani MK, Takallou L, Roshan NM. CD10 expression in gastric carcinoma is correlated with tumor grade and survival. Univ Med. 2019;38(1):41-7. doi: http://dx.doi.org/10.18051.

2. Jacobs J, Smits E, Lardon F, Pauwels P, Deschoolmeester V. Immune Checkpoint Modulation in Colorectal Cancer: What's New and What to Expect. J Immunol Res. 2015;2015:1-16. doi: 10.1155/2015/158038. [PubMed: 26605342].

3. Torre LA, Bray F, Siegel RL, Ferlay J, Lortet-Tieulent J, Jemal A, et al. Global cancer statistics, 2012. CA Cancer I Clin. 2015; 65(2):87-108. doi: 10.3322/caac.21262. [PubMed: 25651787].

4. van de Velde CJ. Surgery: palliative primary tumour resection in mCRC-debate continues. Nat Rev Clin Oncol. 2015; 12(3):129-30. doi: 10.1038/nrclinonc.2015.7. [PubMed: 25601445].

5. Nikiteas NI, Tzanakis N, Gazouli M, Rallis G, Daniilidis K, Theodoropoulos G, et al. Serum IL-6, TNFalpha and CRP levels in Greek colorectal cancer patients: prognostic implications. World J Gastroenterol. 2005;11(11):1639-43. doi: 10.3748/wjg.v11.i11.1639. [PubMed: 15786541].

6. Miki C, Konishi N, Ojima E, Hatada T, Inoue Y, Kusunoki M, et al. C-reactive protein as a prognostic variable that reflects uncontrolled up-regulation of the IL-1-IL-6 network system in colorectal carcinoma. Dig Dis Sci. 2004;49(6):970-6. doi: 10.1023/b:ddas.0000034556.48527.6e. [PubMed: 15309885].

7. Berindan-Neagoe I, Monroig PC, Pasculli B, Calin GA. MicroRNAome genome: a treasure for cancer diagnosis and therapy. CA Cancer J Clin. 2014;64(5):311-36. doi: 10.3322/caac.21244. [PubMed: 25104502].

8. Rupaimoole R, Slack FJ. MicroRNA therapeutics: towards a new era for the management of cancer and other diseases. Nat Rev Drug Discov. 2017;16(3):203-22. doi: 10.1038/nrd.2016.246. [PubMed: 28209991].

9. Zhang G, Liu X, Li Y, Wang Y, Liang H, Li K, et al. EphB3targeted regulation of miR-149 in the migration and invasion of human colonic carcinoma HCT116 and SW620 cells. Cancer Sci. 2017;108(3):408-18. doi: 10.1111/cas.13161. [PubMed: $28370854]$.

10. Zhou W, Wang L, Miao Y, Xing R. Novel long noncoding RNA GACAT3 promotes colorectal cancer cell proliferation, invasion, and migration through miR-149. Onco Targets Ther. 2018;11:1543-52. doi: 10.2147/OTT.S144103.

11. Ravasco P, Monteiro-Grillo I, Camilo M. Individualized nutrition intervention is of major benefit to colorectal cancer patients: long-term follow-up of a randomized controlled trial of nutritional therapy. Am J Clin Nutr. 2012;96(6):1346-53. doi: 10.3945/ajcn.111.018838. [PubMed: 23134880].

12. Mohammadi A, Mansoori B, Baradaran B. The role of microRNAs in colorectal cancer. Biomed Pharmacother.
2016;84:705-13. doi: 10.1016/j.biopha.2016.09.099. [PubMed: 27701052].

13. Link A, Balaguer F, Shen Y, Nagasaka T, Lozano JJ, Boland CR, et al. Fecal MicroRNAs as novel biomarkers for colon cancer screening. Cancer Epidemiol Biomarkers Prev. 2010;19(7): 1766-74. doi: 10.1158/1055-9965.EPI-10-0027. [PubMed: 20551304].

14. Wu CW, Ng SS, Dong YJ, Ng SC, Leung WW, Lee CW, et al. Detection of miR-92a and miR-21 in stool samples as potential screening biomarkers for colorectal cancer and polyps. Gut. 2012;61(5):739-45. doi: 10.1136/gut.2011.239236. [PubMed: 21930727].

15. Lv ZC, Fan YS, Chen HB, Zhao DW. Investigation of microRNA155 as a serum diagnostic and prognostic biomarker for colorectal cancer. Tumour Biol. 2015;36(3):1619-25. doi: 10.1007/s13277-014-2760-9. [PubMed: 25528214].

16. Luo G, Chao YL, Tang B, Li BS, Xiao YF, Xie R, et al. miR-149 represses metastasis of hepatocellular carcinoma by targeting actin-regulatory proteins PPM1F. Oncotarget. 2015;6(35): 37808-23. doi: 10.18632/oncotarget.5676. [Pubmed: 26498692].

17. Jin L, Li Y, Liu J, Yang S, Gui Y, Mao X, et al. Tumor suppressor miR-149-5p is associated with cellular migration, proliferation and apoptosis in renal cell carcinoma. Mol Med Rep. 2016;13(6):5386-92. doi: 10.3892/mmr.2016.5205. [PubMed: 27121091].

18. Yang D, Du G, Xu A, Xi X, Li D. Expression of miR-149-3p inhibits proliferation, migration, and invasion of bladder cancer by targeting S100A4. Am J Cancer Res. 2017; 7(11):2209-19. [PubMed: 29218245].

19. Xu K, Liu X, Mao X, Xue L, Wang R, Chen L, et al. MicroRNA-149 suppresses colorectal cancer cell migration and invasion by directly targeting forkhead box transcription factor FOXM1. Cell Physiol Biochem. 2015;35(2):499-515. doi: 10.1159/000369715. [PubMed: 25613903].

20. Nozoe T, Kimura Y, Ishida M, Saeki H, Korenaga D, Sugimachi K, et al. Correlation of pre-operative nutritional condition with post-operative complications in surgical treatment for oesophageal carcinoma. Eur J Surg Oncol. 2002;28(4):396-400. doi: 10.1053/ejso.2002.1257. [PubMed: 12099649].

21. Watanabe M, Iwatsuki M, Iwagami S, Ishimoto T, Baba Y, Baba $\mathrm{H}$, et al. Prognostic nutritional index predicts outcomes of gastrectomy in the elderly. World J Surg. 2012;36(7):1632-9. doi: 10.1007/s00268-012-1526-z. [PubMed: 22407085].

22. Sorensen J, Kondrup J, Prokopowicz J, Schiesser M, Krahenbuhl L, Meier R, et al. Euro00PS: an international, multicentre study to implement nutritional risk screening and evaluate clinical outcome. Clin Nutr. 2008;27(3):340-9. doi: 10.1016/j.clnu.2008.03.012. [PubMed: 18504063].

23. Schiesser M, Muller S, Kirchhoff $P$, Breitenstein S, Schafer M, Clavien PA, et al. Assessment of a novel screening score for nutritional risk in predicting complications in gastrointestinal surgery. Clin Nutr. 2008;27(4):565-70. doi: 10.1016/j.clnu.2008.01.010. [PubMed: 18342995].

24. Wang Y, Zheng J, Gao Z, Han X, Qiu F. Investigation on nutritional risk assessment and nutritional support status of surgical patients with colorectal cancer. J Buon. 2018;23(1):62-7. [PubMed: 29552761]. 